\title{
PROGRESS IN HIGHER SPIN GAUGE THEORIES
}

\author{
M.A.VASILIEV ${ }^{1}$ \\ ${ }^{1}$ I.E.Tamm Department of Theoretical Physics, Lebedev Physical Institute, \\ Leninsky prospect 53, 117924, Moscow, Russia
}

\begin{abstract}
General properties of the theory of higher spin gauge fields in $A d S_{4}$ are discussed. Some new results on the $4 \mathrm{~d}$ conformal higher spin gauge theory are announced. Talk given at the International Conference "Quantization, Gauge Theory and Strings" in memory of E.S.Fradkin, Moscow, June 5-10, 2000.
\end{abstract}

\section{Introduction}

Higher spin theory is a classical subject of the field theory. It takes origin from the works of Dirac [1], Fierz and Pauli [2], Rarita and Schwinger [3] and many others. It is also a traditional subject in the Theory Department of the Lebedev Physical Institute where Professor Efim Samoilovich Fradkin was working most of his scientific life and where this problematics was initiated by Tamm and Ginzburg [4] (see [5] for more historical remarks and references). In fact, Fradkin got a position at the Theory Department by recommendation of Vitaly Lazarevich Ginzburg who liked the investigation of Efim Samoilovich on the theory of spin $5 / 2$ field, the subject of his first scientific paper [6].

The concept of higher spins is time-dependent. For about forty years the main interest was focused on the massive fields and "higher spin" (HS) meant $s \geq 3 / 2$ (sometimes, even $s=1$ ). The situation changed after creation of supergravity in 1976 [7, 8]. One possible interpretation of supergravity is that it results from resolution of the problem of consistent interactions for the massless spin $3 / 2$ field. A lesson was that the principle of gauge symmetry associated with the corresponding massless field (local supersymmetry for $s=$ 3/2) played a key role in the theory. As a result, since 1976 the interest shifted to the massless fields associated with gauge symmetries with the updated convention that "higher spin" implies $s \geq 5 / 2$.

In the early seventies Efim Samoilovich was mainly interested in the HS theory as a range for the application of the new methods of quantization of constrained systems he was working on. As an undergraduate student of Efim Samoilovich I was lucky to get a problem related to the Hamiltonian analysis of the spin $3 / 2$ and spin 2 massive fields in external fields thus learning both 
the constrained dynamics and the HS theory. However, one day in spring of 1976 my research plans have changed drastically after I got a phone call from Efim Samoilovich with the urgent instruction to go to the Moscow international airport Sheremet'evo to get a copy of some new important paper from Vladimir Kadyshevsky coming from CERN. Needless to say that those days it was the most efficient way of mailing news to USSR. What I got was the first paper on supergravity by Ferrara, Freedman and van Nieuwenhuizen [7]. This case illustrates perfectly how precise was the feeling of Efim Samoilovich of what is really important in science.

Supergravity became one of the main scientific interests of Efim Samoilovich. A development having particular importance for the $\mathrm{HS}$ problem was $\mathrm{N}=2$ gauged supergravity found independently be Freedman and Das [9] and by us [10. What we learned from this model was that at certain circumstances (particularly, if the gauge coupling constant between gravitino and vector fields in the supergravity supermultiplet is non-zero) the gauge symmetry principle may require non-zero cosmological constant.

In April 1978, when the original enthusiasm on the possibility to build a complete fundamental theory in the framework of supergravity changed to skepticism, we arrived at the idea to generalize supergravity to higher spins (in particular, to spin 5/2). It is well known that massless fields of spin $1\left(A_{n}\right)$, $3 / 2\left(\psi_{n \alpha}\right)$ and spin $2\left(g_{n m}\right)$ are gauge fields with the transformation laws

$$
\delta A_{n}=\partial_{n} \varepsilon, \quad \delta \psi_{n \alpha}=\partial_{n} \varepsilon_{\alpha}, \quad \delta g_{n m}=\partial_{n} \varepsilon_{m}+\partial_{m} \varepsilon_{n} .
$$

( $m, n \ldots$ are vector indices and $\alpha, \beta \ldots$ are spinor indices). The situation with HS massless fields is analogous. As was first shown by Fronsdal [11], an integerspin massless spin $-s$ gauge field is described by the totally symmetric tensor $\varphi_{n_{1} \ldots n_{s}}$ subject to the double tracelessness condition [1] $\varphi_{r}^{r}{ }_{r}^{s}{ }_{s n_{5} \ldots n_{s}}=0$ which is nontrivial for $s \geq 4$. A quadratic action [11] for a free spin $s$ field $\varphi_{n_{1} \ldots n_{s}}$ is fixed unambiguously [12] (up to an overall factor) in the form $S_{s}=\varphi L \varphi$ with some second order differential operator $L$ by the requirement of gauge invariance under the Abelian gauge transformations

$$
\delta \varphi_{n_{1} \ldots n_{s}}=\partial_{\left\{n_{1}\right.} \varepsilon_{\left.n_{2} \ldots n_{s}\right\}}
$$

with the parameters $\varepsilon_{n_{1} \ldots n_{s-1}}$ which are rank- $(s-1)$ totally symmetric traceless tensors, $\varepsilon_{r n_{3} \ldots n_{s-1}}^{r}=0$. This formulation is parallel [14] to the metric formulation of gravity and is called formalism of symmetric tensors. Fermionic HS gauge fields are described analogously [13] in terms of rank- $(s-1 / 2)$ totally symmetric spinor-tensors $\psi_{n_{1} \ldots n_{s-1 / 2} \alpha}$ subject to the $\gamma$-tracelessness condition $\gamma_{\alpha}^{s}{ }_{\alpha}^{\beta} \psi_{r s n_{4} \ldots n_{s-1 / 2} \beta}^{r}=0$. 
The problem was to introduce interactions of HS fields with some other fields in a way compatible with HS gauge symmetries. A particularly important example is provided by the interaction with gravity. It was straightforward to see that the standard covariantization procedure $\partial \rightarrow D=\partial-\Gamma$ breaks down the invariance under the HS gauge transformations because it turns out that, in order to prove invariance of the action $S_{s}$, one has to commute derivatives, while the commutator of the covariant derivatives is proportional to the Riemann tensor, $[D \ldots, D \ldots]=\mathcal{R} \ldots$. As a result, the gauge variation of the covariantized action $S_{s}^{c o v}$ has the following structure:

$$
\delta S_{s}^{c o v}=\mathcal{R}_{\ldots}\left(\varepsilon_{\ldots} D \varphi_{\ldots}\right) \neq 0 .
$$

It was not clear what to do with these terms because for $s>2$ they contain the Weyl part of the Riemann tensor that cannot be compensated by any transformation of the gravitational field [15]. However, having learnt the role of the cosmological constant in the gauged supergravity, it was natural to reconsider the problem in the AdS background. (As a result, our study was not affected by the papers on the difficulties of the gravitational interaction of HS fields [15], all assuming implicitly an expansion near the flat space.) Somewhat later this led to the desired result as was originally checked for spin 3 by a rather complicated straightforward analysis [16]. A number of interesting papers on the existence of some consistent interactions of the HS fields appeared in eighties both in the light-cone approach [17 and in the covariant approach [18], providing a strong evidence that some fundamental HS gauge theory must exist. However, in all these works, the problem was considered in the flat space and therefore no progress with the gravitational interaction was achieved.

The key point of the relevance of the AdS background is as follows. Near the AdS background, the Riemann tensor $\mathcal{R}$ is not small but $\mathcal{R}=R+\lambda^{2} g g$, where $\lambda^{-1}$ is the radius of the AdS space, $g$ is the background AdS metric tensor and $R$ denotes a fluctuation of the Riemann tensor near the background curvature. When expanding around the AdS geometry one therefore has to expand in powers of $R$ rather than in powers of the Riemann tensor itself. In other words, in the AdS space the commutator of covariant derivatives is of order $\lambda^{2}$. Now one can modify the action by adding some cubic terms of the form

$$
S^{\text {int }}=\int_{M_{4}} \sum_{A, B} \lambda^{-(A+B)} D^{A}(\phi) D^{B}(\phi) R
$$

containing higher derivatives of the dynamical fields with the coefficients proportional to negative powers of $\lambda$. Note that for any two given spins a highest 
order of the derivatives in a vertex is finite increasing linearly with the sum of spins. As we checked originally for the case of spin 3 [16], there exists such a unique (modulo total derivatives and field redefinitions) action (4) that its HS gauge variation exactly compensates the original variation (3).

The fundamental concept of the HS gauge theory is the underlying symmetry principle. By construction, the class of HS gauge theories consists of most symmetric theories having as many as possible symmetries unbroken (any more symmetric theory will have more lower and/or higher spin symmetries and therefore will belong to the class of HS theories). As such HS gauge theory is of particular importance for the search of a fundamental symmetric phase of the superstring theory. This is most obvious in the context of the so called Stueckelberg symmetries in the string field theory which have a form of some spontaneously broken HS gauge symmetries. Whatever a symmetric phase of the superstring theory is, Stueckelberg symmetries are expected to become unbroken HS symmetries in such a phase and, therefore, the superstring field theory has to become one or another version of the HS gauge theory. One explanation why the HS gauge theory has not been yet observed in the superstring theory is that still no complete formulation of the latter is known in the AdS background.

To elucidate a structure of the HS algebra we used the so-called "geometric" approach to supergravity [21, 22] in which fields, action and transformation laws are formulated in terms of the gauge fields of some (super)algebra identified with the global symmetry algebra of a theory. An attractive feature of this machinery is that it avoids explicit contraction of indices by the metric tensor, treating all fields as differential forms. The distinguishing property of the metric tensor that appears on equal footing with other massless fields in a HS multiplet is that it has a nonzero vacuum expectation value providing a meaningful linearized approximation for all fields in the model.

As a first step towards such a formulation it was instructive to check whether it is possible to reformulate the free dynamics of the HS fields in terms of some curvatures linear in the dynamical fields and containing the background (Poincare or AdS) gravitational vierbein and Lorentz connection 1 -forms. As the lowest nontrivial dimension with propagating HS fields is $\mathrm{d}=4$, we first focused on this case. It was shown that such free actions do indeed exist 23. (the generalization to any dimension was later given in 24 thus indicating that the same approach is likely to be successful in any $d$ ). The resulting formulation of the free field HS dynamics is a generalization of the Cartan (frame) formulation of gravity. Assuming that thus found linearized HS curvatures result from the linearization of some non-Abelian HS curva- 
tures, one deduces from their form some of the structure coefficients of the full HS algebra (namely those that correspond to the commutators of any generator with the generators of the space-time algebra (Poincare or AdS) giving rise to the gravitational gauge fields). These data fix (up to a multiplicity) a possible pattern of the reduction of the full algebra with respect to its spacetime subalgebra and can be used as a sort of "initial data" for the problem of reconstruction of the full non-Abelian HS algebra. Adding some (more or less) natural assumptions on the structure of the HS algebra, its structure coefficients were found explicitly in [19] by a direct solution of the Jacobi identities. A few years later it was observed [20] that this algebra admits a simple realization in terms of the oscillator (i.e., star product) algebra with spinor generating elements. As we demonstrate in this talk this fact links together such seemingly different properties of the HS theories as the relevance of the AdS background, necessity of introducing infinitely many spins in the HS models and space-time non-locality of the HS interactions manifested by the formula (14). Note that altogether these properties make the HS theories reminiscent of the superstring theory with the analogy between the cosmological constant and the parameter $\alpha^{\prime}$. Remarkably, the properties of the HS theory that follow from the structure of the HS algebra found in eighties [19, 20] "predicted" some of the present day hot topics in the superstring theory.

\section{$2 \quad \mathbf{D}=4$ Higher Spin Algebra and $A d S$ Vac- uum}

Perturbative HS dynamics is formulated in terms of the "superfield" 1-form $\omega(Y \mid x)=d x^{\underline{n}} \omega_{\underline{n}}(Y \mid x)$ and 0-form $C(Y \mid x)$ that describe, respectively, the HS gauge fields and (HS) Weyl tensors along with matter fields. They depend on the space-time coordinates $x^{\underline{n}}$ and commuting Majorana spinors $Y_{\Omega}=\left(y_{\alpha}, \bar{y}_{\dot{\alpha}}\right)$ $(\Omega, \Lambda \ldots=1 \div 4 ; \alpha, \beta=1,2 ; \dot{\alpha}, \dot{\beta}=1,2)$. The difference with the standard superfield approach is that the additional spinor coordinates $Y_{\Omega}$ commute and therefore the HS superfields contain infinite chains of the space-time component fields in their expansion in power series in the spinor variables

$$
\begin{gathered}
\omega(y, \bar{y} \mid x)=\sum_{n, m=0}^{\infty} \frac{1}{2 i m ! n !} d x^{l} \omega_{\underline{l}}^{\alpha_{1} \ldots \alpha_{n} \dot{\alpha}_{1} \ldots \dot{\alpha}_{m}}(x) y_{\alpha_{1}} \ldots y_{\alpha_{n}} \bar{y}_{\dot{\alpha}_{1}} \ldots \bar{y}_{\dot{\alpha}_{m}}, \\
C(y, \bar{y} \mid x)=\sum_{n, m=0}^{\infty} \frac{1}{2 i m ! n !} C^{\alpha_{1} \ldots \alpha_{n} \dot{\alpha}_{1} \ldots \dot{\alpha}_{m}}(x) y_{\alpha_{1}} \ldots y_{\alpha_{n}} \bar{y}_{\dot{\alpha}_{1}} \ldots \bar{y}_{\dot{\alpha}_{m}}
\end{gathered}
$$


Here the component fields carrying (odd) even numbers of spinor indices are required to be (anti)commutingt. All the component fields $\omega_{\underline{n}}^{\alpha_{1} \ldots \alpha_{n} \dot{\alpha}_{1} \ldots \dot{\alpha}_{m}}(x)$ with $n+m=2(s-1)$ and $C^{\alpha_{1} \ldots \alpha_{n} \dot{\alpha}_{1} \ldots \dot{\alpha}_{m}}(x)$ with $|n-m|=s$ are associated with the spin $s$. The dynamical spin $s \geq 1$ fields are identified with $\omega_{\underline{n}}^{\alpha_{1} \ldots \alpha_{n} \dot{\alpha}_{1} \ldots \dot{\alpha}_{m}}(x)$ at $|n-m|=0$ for bosons and $|n-m|=1$ for fermions. The matter fields with $s=0$ and $1 / 2$ are identified with $C^{\alpha_{1} \ldots \alpha_{n} \dot{\alpha}_{1} \ldots \dot{\alpha}_{m}}(x)$ at $n=m=0$ and $n+m=1 / 2$, respectively. All other fields express via higher derivatives of the dynamical fields by virtue of appropriate constraints [23].

The 1-forms $\omega(Y \mid x)$ are gauge fields of the HS algebra. The HS field strength has the standard form $R^{A}=d \omega^{A}+f_{B C}^{A} \omega^{B} \wedge \omega^{C}$ where $f_{B C}^{A}$ are the structure coefficients of the HS algebra originally found in [19]. As shown in [20] these HS curvatures result from the star product construction

$$
R(Y \mid x)=d \omega(Y \mid x)-(\omega \wedge * \omega)(Y \mid x),
$$

where the space-time coordinates $x^{\underline{n}}$ are commuting while the star product acts on the auxiliary coordinates $Y$ according to the rule

$$
\begin{aligned}
(f * g)(Y) & =\frac{1}{(2 \pi)^{2}} \int d^{4} U d^{4} V \exp \left(i U_{\Omega} V^{\Omega}\right) f(Y+U) g(Y+V) \\
& =\left.e^{i \frac{\partial}{\partial Y_{\Omega}^{1}} \frac{\partial}{Y^{2 \Omega}}} f\left(Y+Y^{1}\right) B\left(Y+Y^{2}\right)\right|_{Y^{1}=Y^{2}=0},
\end{aligned}
$$

where $U_{\Omega} V^{\Omega}=U_{\Omega} V_{\Omega^{\prime}} C^{\Omega \Omega^{\prime}}$ and $C_{\Omega \Omega^{\prime}}$ is the $4 \mathrm{~d}$ charge conjugation matrix used to raise and lower spinor indices $U^{\Omega}=C^{\Omega \Omega^{\prime}} U_{\Omega^{\prime}}, U_{\Omega}=U^{\Omega^{\prime}} C_{\Omega^{\prime} \Omega}$. This star product defines the associative algebra $A_{4}$ (called Weyl algebra) with the defining relation $Y_{\Omega} * Y_{\Omega^{\prime}}-Y_{\Omega^{\prime}} * Y_{\Omega}=2 i C_{\Omega \Omega^{\prime}}$. It describes the product of Weyl ordered (i.e. totally symmetric) polynomials of oscillators in terms of symbols of operators [25]. This Weyl product law (called Moyal bracket 26] for commutators constructed from (8)) is obviously nonlocal manifesting the ordinary quantum-mechanical nonlocality.

The 0-form $C(Y \mid x)$ belongs to the twisted adjoint representation with the covariant derivative

$$
D C(Y \mid x)=d C(Y \mid x)-(\omega * C)(Y \mid x)+(C * \tilde{\omega})(Y \mid x),
$$

\footnotetext{
${ }^{1}$ To make the relationship between spin and statistics more obvious it is convenient to assume that $\omega$ and $C$ depend on an additional Clifford element $\psi\left(\psi^{2}=1\right)$ requiring the fields $\omega(\psi, Y \mid x)$ and $C(\psi, Y \mid x)$ to be even functions of the auxiliary variables, i.e. $\omega(\psi, Y \mid x)=$ $\omega(-\psi,-Y \mid x)$ and $C(\psi, Y \mid x)=C(-\psi,-Y \mid x)$. Obviously, $\psi$ merely labels fermions and can be discarded as in (5) and (6). This construction is particularly useful however in the HS models with extended supersymmetry resulting [20] from introducing a number of Clifford elements $\psi^{i}, i=1 \div N$.
} 
where $\sim$ is the chirality flipping automorphism of the algebra

$$
\tilde{f}(y, \bar{y})=f(y,-\bar{y}) .
$$

Note that in the bosonic case with $f(-y,-\bar{y})=f(y, \bar{y})$ there is no difference between changing a sign of either $y$ or $\bar{y}$. In the fermionic case the two cases are not equivalent and, for the complete formulation, it is necessary to double the fields as discussed in the section 5 .

The HS gauge transformations are

$$
\begin{aligned}
& \delta \omega(Y \mid x)=d \epsilon(Y \mid x)-(\omega * \epsilon)(Y \mid x)+(\epsilon * \omega)(Y \mid x), \\
& \delta C(Y \mid x)=(\epsilon * C)(Y \mid x)-(C * \tilde{\epsilon})(Y \mid x) .
\end{aligned}
$$

Since, as explained in the section 4), higher components in the expansions of $\omega(Y \mid x)$ and $C(Y \mid x)$ in powers of $Y$ identify with the higher derivatives of the dynamical HS fields by virtue of constraints, the field transformation law effectively contains higher space-time derivatives of the dynamical fields in the terms containing higher components of the gauge parameters $\epsilon(Y \mid x)$. Thus, the quantum-mechanical nonlocality in the auxiliary spinor coordinates $Y_{\Omega}$ induces the space-time derivatives of all orders in the HS transformation laws. This is in accordance with the fact [27, 28] that a spin $s$ conserved current $T_{n_{1} \ldots n_{s}} \sim \partial_{n_{1}} \ldots \partial_{n_{l}} \bar{\phi} \partial_{n_{l}+1} \ldots \partial_{n_{s}} \phi$ contains derivatives of order $s$.

A structure of the full nonlinear HS equations of motion is such that any solution $\omega_{0}$ of the zero-curvature equation

$$
d \omega=\omega * \wedge \omega
$$

solves the HS equations. Locally, such a vacuum solution admits a pure gauge form $\omega_{0}=-g^{-1}(Y \mid x) * d g(Y \mid x)$ with some invertible element $g(Y \mid x)$ of the Weyl algebra, $g * g^{-1}=g^{-1} * g=I$. It breaks the local HS symmetry to its stability subalgebra with the infinitesimal parameters $\epsilon_{0}(Y \mid x)$ satisfying the equation $D_{0} \epsilon_{0} \equiv d \epsilon_{0}-w_{0} * \epsilon_{0}+\epsilon_{0} * w_{0}=0$ which solves as $\epsilon_{0}(Y \mid x)=$ $g^{-1}(Y \mid x) * \epsilon_{0}(Y) * g(Y \mid x)$. In the HS theories no further symmetry breaking is induced by the field equations, i.e. $\epsilon_{0}(Y)$ parametrizes the global symmetry of the theory. Therefore, the HS global symmetry algebra identifies with the Lie superalgebra constructed from the (anti)commutators of the elements of the Weyl algebra. Note that the fields carrying odd numbers of spinor indices are anticommuting thus inducing the superalgebra structure into (12).

Functions bilinear in $Y_{\Omega}$ form a subalgebra with respect to star-commutators. This is the $A d S_{4}$ algebra $s p(4 ; R) \sim o(3,2)$. One can look for a solution of the 
vacuum equation (12) in the form

$$
\omega_{0}=\frac{1}{4 i}\left(\omega_{0}^{\alpha \beta}(x) y_{\alpha} y_{\beta}+\bar{\omega}_{0}^{\dot{\alpha} \dot{\beta}}(x) \bar{y}_{\dot{\alpha}} \bar{y}_{\dot{\beta}}+2 \lambda h_{0}^{\alpha \dot{\beta}}(x) y_{\alpha} \bar{y}_{\dot{\beta}}\right) .
$$

Inserting this formula into (12) one finds that the fields $\omega_{0}, \bar{\omega}_{0}$ and $h_{0}$ identify, respectively, with the Lorentz connection and the frame field of $A d S_{4}$ provided that the frame $h_{0}$ is invertible. The parameter $\lambda=r^{-1}$ is identified with the inverse AdS radius. Thus, the star product origin of the HS algebra leads to the AdS geometry as a natural vacuum solution.

Note that spin $s>2$ gauge fields are described by the degree $2(s-1)$ polynomials which do not close to any finite-dimensional subalgebra. Therefore, HS $(s>2)$ massless fields can only appear in infinite sets of massless fields with infinitely increasing spins. In other words, any finite-dimensional subalgebra of the HS symmetry algebra containing the Lorentz subalgebra can have at most spin 2 gauge fields. One can speculate on some spontaneous breakdown of the HS symmetries down to a finite-dimensional subalgebra (followed by a flat contraction via a shift of the vacuum energy in the broken phase).

In a physical phase with $\lambda \sim 0$ and $m \gg m^{\exp }$ for HS fields, only usual sets of lower spin gauge fields can remain massless.

\section{Higher Spin Action in $A d S_{4}$}

The HS action used in [29] is formulated in terms of the components of the curvatures (7)

$$
R(y, \bar{y} \mid x)=\sum_{n, m=0}^{\infty} \frac{1}{2 i m ! n !} R^{\alpha_{1} \ldots \alpha_{n} \dot{\alpha}_{1} \ldots \dot{\alpha}_{m}}(x) y_{\alpha_{1}} \ldots y_{\alpha_{n}} \bar{y}_{\dot{\alpha}_{1}} \ldots \bar{y}_{\dot{\alpha}_{m}}
$$

as follows

$$
S=-\frac{1}{4 \kappa^{2} \lambda^{2}} \sum_{m, n=0}^{\infty} \frac{i^{n+m-1}}{n ! m !} \epsilon(n-m) \int_{M^{4}} R_{\alpha_{1} \ldots \alpha_{n} \dot{\beta}_{1} \ldots \dot{\beta}_{m}} \wedge R^{\alpha_{1} \ldots \alpha_{n} \dot{\beta}_{1} \ldots \dot{\beta_{m}}},
$$

where $\epsilon(n)$ is a sign function, i.e. $\epsilon(0)=0$ and $\epsilon( \pm n)= \pm 1$ for any positive integer $n$. To analyze this action perturbatively one expands the gauge fields as $\omega=\omega_{0}+\omega_{1}$, where $\omega_{0}$ is a zero curvature vacuum solution (i.e. $R_{0}=0$ ) and $\omega_{1}$ is the dynamical part. In what follows we identify a vacuum solution with some $A d S_{4}$ fields of the form (13). The HS curvatures then expand as $R=R_{1}+R_{2}$, where

$$
R_{1}(Y \mid x)=d \omega_{1}(Y \mid x)-\left(\omega_{0} * \wedge \omega_{1}\right)(Y \mid x)-\left(\omega_{1} * \wedge \omega_{0}\right)(Y \mid x) .
$$


The quadratic part of the action has the form (15) with $R_{1}$ instead of $R$. It was originally found in [23] along with the form of the linearized curvatures from the condition that its variation with respect to the "extra fields" $\omega_{\alpha_{1} \ldots \alpha_{n} \dot{\beta}_{1} \ldots \dot{\beta}_{m}}$ with $|n-m|>2$ vanishes identically. The variation with respect to the dynamical fields, $\omega_{\alpha_{1} \ldots \alpha_{n} \dot{\beta}_{1} \ldots \dot{\beta}_{m}}$ with $|n-m| \leq 2$ remains non-trivial and leads to the correct free equations for a spin $s \geq 3 / 2$ massless field in the sector of $n+m=2(s-1)$.

Beyond the quadratic order, the extra fields do contribute into the interaction terms. One therefore has to express them in terms of the dynamical fields to have a well-defined nonlinear action. The appropriate linearized constraints have the form 23]

$$
h_{\{\alpha}^{\dot{\gamma}} \wedge R_{\left.1 \alpha_{1} \ldots \alpha_{n}\right\} \dot{\beta_{1} \ldots \beta_{m} \dot{\gamma}}}=0 \quad(n>m \geq 0)
$$

(and complex conjugate). These constraints express algebraically all extra fields $\omega_{\alpha_{1} \ldots \alpha_{n} \dot{\beta}_{1} \ldots \dot{\beta}_{m}}$ with $|n-m|>2$ in terms of the dynamical fields $\omega_{\alpha_{1} \ldots \alpha_{n} \dot{\beta}_{1} \ldots \dot{\beta}_{m}}$ with $|n-m| \leq 2$ and their derivatives. They play a key role in the description of the HS dynamics, governing a form of the interactions of the dynamical HS fields in the action (15) and, in particular, giving rise to higher derivatives and negative powers of the cosmological constant in the HS interactions.

Remarkably, the action (15) supplemented with the constraints (17) turns out to be gauge invariant under (appropriately deformed) HS symmetries 29] up to $o\left(\phi^{2}\right) \epsilon$ terms (note that this requirement fixes uniquely the relative coefficients in front of the individual spin- $s$ quadratic actions in (15)). This action is explicitly general coordinate invariant and reduces to the Einstein-Hilbert action in the MacDowell-Mansouri form [22] in the spin-2 sector $(n+m=2)$. Therefore, it solves the original problem of a higher-spin-gravitational interaction at the cubic order. Let us note that the described approach leads to a particular form of the action (1) but avoids complicated computations and is uncomparably simpler than the straightforward analysis [16].

A non-trivial question is how to extend this result to the highest orders in interactions. To proceed one has first of all to determine the full spectrum of fields in the theory . Another problem is to find an appropriate generalization of the linearized constraints (17). This program was completed at the level of the equations of motion [30] (see also [28]) in terms of appropriate generating functions of the auxiliary spinor variables satisfying certain flatness conditions

\footnotetext{
${ }^{2}$ Note that a reduction of a full consistent action by setting any subset of fields equal to zero still leads to an action consistent at the cubic order (as is most obvious from its Noether current character) even in case this reduction cannot be consistently extended beyond the cubic approximation.
} 
(this formalism is referred to as "unfolded formulation"). As a result, the full answer to the question on the true spectra of HS and lower spin fields compatible with the consistent interactions was given and the full nonlinear system of constraints and equations of motion was formulated. These results are summarized in the section 5 .

Let us mention that an alternative fruitful approach towards HS gauge theory into which Fradkin and Metsaev contributed a lot 31] is the light-cone analysis of the problem initiated in [17]. It is complementary to the covariant approach: the latter makes all symmetries explicit by virtue of introducing infinite sets of auxiliary fields, while the former breaks down all HS gauge symmetries to formulate dynamics directly in terms of the dynamical degrees of freedom. Another important direction in the HS gauge theory developed by Fradkin and Linetsky is conformal HS gauge theory [32, 33]. Although, analogously to the case of conformal (super)gravity (for more details and references on conformal supergravity see [34]), it is not unitary in $d=4$ (i.e., contains ghosts) conformal HS gauge theory can lead to a compact formulation of the unitary (AdS) HS theory by virtue of introducing compensators. In the section 6 we will announce some new result on the unfolded formulation of the linearized conformal 4d HS theories that, hopefully, provides a good starting point to study a full nonlinear conformal HS gauge theory.

\section{Unfolded Form of the Free HS Equations}

From the constraints (17) along with the free equations of motion that follow from the linearized action (15) one proves by virtue of the Bianchi identities [23] that

$$
R_{1}(y, \bar{y} \mid x)=h^{\gamma \dot{\beta}} \wedge h_{\gamma}{ }^{\dot{\alpha}} \frac{\partial}{\partial \bar{y}^{\dot{\alpha}}} \frac{\partial}{\partial \bar{y}^{\dot{\beta}}} C(0, \bar{y} \mid x)+h^{\alpha \dot{\gamma}} \wedge h^{\beta} \dot{\gamma} \frac{\partial}{\partial y^{\alpha}} \frac{\partial}{\partial y^{\beta}} C(y, 0 \mid x),
$$

i.e. most of the components of the HS curvatures vanish on-mass-shell except for those parametrized by the holomorphic $(C(y, 0 \mid x))$ and antiholomorphic $(C(0, \bar{y} \mid x))$ parts of $C(Y \mid x)$. For spin 2 these are order-4 polynomials in $y$ or $\bar{y}$ parametrizing the Weyl tensor. For higher spins, $C(y, 0 \mid x)$ and $C(0, \bar{y} \mid x)$ parametrize the "HS Weyl tensors". The equation (18) is an alternative form of the free HS field equations equivalent to the standard one.

By virtue of the Bianchi identities, the equation (18) imposes some differ-

ential conditions on the Weyl tensors. As shown in [35], for spins $s \geq 3 / 2$ 
these differential conditions turn out to be equivalent to the equation

$$
D_{0} C \equiv d C-w_{0} * C+C * \tilde{w}_{0}=0
$$

where $D_{0}$ is the linearized covariant derivative (9). For the $s=1$ case, the equation (18) does not impose any restrictions on the holomorphic and antiholomorphic 0-forms $C(Y \mid x)$ bilinear in $Y$ that parametrize the spin 1 field strength. The Maxwell equation is contained in (19). For the cases of spin 0 and spin 1/2 matter fields there are no associated gauge fields and the respective (Klein-Gordon and Dirac) equations are also contained in (19). To summarize, the content of the equations (18), (19) is twofold: they describe usual free field equations for all spins and express all extra gauge fields and 0-forms $C(Y \mid x)$ in terms of the higher derivatives of the dynamical fields.

Since changes a sign of the $A d S_{4}$ translations thus transforming the commutator in the adjoint representation into the anticommutator in (9) for the terms linear in $h, D_{0}$ in (19) has the form $D_{0} C(y, \bar{y} \mid x) \equiv D^{L} C(y, \bar{y} \mid x)+$ $\frac{i \lambda}{2}\left\{h^{\alpha \dot{\beta}} y_{\alpha} \bar{y}_{\dot{\beta}}, C(y, \bar{y} \mid x)\right\}_{*}$ where $\{a, b\}_{*}=a * b+b * a$ and $D^{L}$ is the Lorentz covariant derivative,

$$
D^{L} C(y, \bar{y} \mid x)=d C(y, \bar{y} \mid x)+\frac{i}{4}\left(\left[\omega^{\alpha \beta} y_{\alpha} y_{\beta}+\bar{\omega}^{\dot{\alpha} \dot{\beta}} \bar{y}_{\dot{\alpha}} \bar{y}_{\dot{\beta}}, C(y, \bar{y} \mid x)\right]_{*}\right) .
$$

By virtue of (8) we get

$$
D_{0} C(y, \bar{y} \mid x) \equiv D^{L} C(y, \bar{y} \mid x)+i \lambda h^{\alpha \dot{\beta}}\left(y_{\alpha} \bar{y}_{\dot{\beta}}-\frac{\partial}{\partial y^{\alpha}} \frac{\partial}{\partial \bar{y}^{\dot{\beta}}}\right) C(y, \bar{y} \mid x)=0 .
$$

From this expression it is clear that (19) links the derivatives in the space-time coordinates $x^{\underline{n}}$ with those in the auxiliary spinor variables $y_{\alpha}$ and $\bar{y}_{\dot{\alpha}}$. Schematically, in the sector of 0 -forms $C(Y \mid x)$, the derivatives in the auxiliary spinor variables form a square root of the space-time derivatives, $\frac{\partial}{\partial x^{\underline{n}}} C(y, \bar{y} \mid x) \sim$ $\lambda \underline{\underline{n}}^{\alpha \dot{\beta}} \frac{\partial}{\partial y^{\alpha}} \frac{\partial}{\partial \bar{y}^{\beta}} C(y, \bar{y} \mid x)$. Explicit component expression is

$$
C_{\alpha_{1} \ldots \alpha_{n}, \dot{\beta}_{1} \ldots \dot{\beta}_{m}}=\frac{1}{(2 i \lambda)^{m}} h_{\alpha_{1} \dot{\beta}_{2}}^{\underline{l}} D_{\underline{l}_{1}}^{L} \ldots h_{\alpha_{m} \dot{\beta}_{m}}^{\underline{\underline{l}}{ }_{m}} D_{\underline{l}_{m}}^{L} C_{\alpha_{m+1} \ldots \alpha_{n}} \quad n \geq m
$$

(and complex conjugated). Analogous formulas are true for the gauge 1-forms [23]. Note that these expressions, as well as those for the extra gauge fields resulting from the resolution of the constraints $(17)$, contain negative powers of $\lambda$. As a result, insertion of the expressions for the extra fields in terms of the dynamical HS fields into the action (15) gives rise to some terms containing 
higher derivatives and negative powers of $\lambda$ in the interaction terms. Note also that the requirement that the free action is independent of the extra fields acquires now a simple interpretation as the condition that the free action has to be free of higher derivatives and regular in the flat limit.

A few comments are now in order.

(i) AdS vacuum geometry implies that the vacuum fields (13) are bilinear in the spinor oscillators and, as a consequence, that the relationship (21) is local, containing at most two derivatives in $Y$. This guarantees that the free field HS dynamics in $A d S_{4}$ is local.

(ii) The nonlocality in $Y$ at the interaction level induced by the star product (8) implies the space-time nonlocality of HS interactions.

(iii) As a consequence of the field equations, the gauge transformation law (11) contains effectively the higher space-time derivatives via higher derivatives in $Y$ induced by the star product. The higher power in $Y$ (i.e., spin) of the HS gauge parameter is, the higher space-time derivatives appear in the transformation law.

(iv) Vacuum solutions in the HS theory different from the most symmetric $A d S_{4}$ one may give rise to nonlocality in the linearized approximation. An interesting problem for the future is to study HS theory in the magnetic background to see whether or not it leads to the same type of nonlocality as in the non-commutative regime in the string theory [36].

Thus, the following facts turn out to be strongly correlated: (i) HS algebras are described by the star product in the auxiliary spinor space; (ii) relevance of the AdS background and (iii) potential space-time nonlocality of the HS interactions due to the appearance of higher derivatives at the nonlinear level.

\section{Nonlinear Higher Spin Equations}

Now we discuss the full nonlinear system of $4 \mathrm{~d}$ HS equations following to [30, 28]. The key element of the construction consists of the extension of the space of auxiliary variables by the doubling of auxiliary Majorana spinor variables $Y_{\Omega}$ in the HS 1-form $\omega(Y \mid x) \longrightarrow W(Z ; Y ; K \mid x)$ and 0 -form $C(Y \mid x) \longrightarrow$ $B(Z ; Y ; K \mid x)$ and by introducing a pair of Klein elements $K=(k, \bar{k})$ having the properties $k^{2}=\bar{k}^{2}=1, \quad k \bar{k}=\bar{k} k$,

$$
\begin{aligned}
& k f\left(z_{\alpha}, \bar{z}_{\dot{\alpha}} ; y_{\alpha}, \bar{y}_{\dot{\alpha}}\right)=f\left(-z_{\alpha}, \bar{z}_{\dot{\alpha}} ;-y_{\alpha}, \bar{y}_{\dot{\alpha}}\right) k \\
& \bar{k} f\left(z_{\alpha}, \bar{z}_{\dot{\alpha}} ; y_{\alpha}, \bar{y}_{\dot{\alpha}}\right)=f\left(z_{\alpha},-\bar{z}_{\dot{\alpha}} ; y_{\alpha},-\bar{y}_{\dot{\alpha}}\right) \bar{k}
\end{aligned}
$$


By definition, $\bar{k}$ generates the automorphism while $k$ generates the conjugated automorphism. The dependence on $K$ leads to the doubling of the HS fields necessary in presence of fermions and also gives rise to some auxiliary fields [35, 28].

The dependence on the additional variables $Z_{\Omega}$ is determined in terms of the "initial data" identified with the HS fields discussed so far

$$
W(0 ; Y ; k, \bar{k} \mid x)=\omega(Y ; k, \bar{k} \mid x), \quad B(0 ; Y ; k, \bar{k} \mid x)=C(Y ; k, \bar{k} \mid x)
$$

by appropriate equations formulated below and effectively describes all nonlinear corrections to the HS field equations. It is convenient to introduce a new compensator-type spinor field $S_{\Omega}(Z ; Y ; Q \mid x)$ which does not carry its own degrees of freedom. It plays a role of a covariant differential along the additional $Z_{\Omega}$ directions. To interpret $S_{\Omega}(Z ; Y ; K \mid x)$ as a $Z-1$-form $S=d Z^{\Omega} S_{\Omega}$ we introduce the anticommuting $Z$-differentials $d Z^{\Omega} d Z^{\Lambda}=-d Z^{\Lambda} d Z^{\Omega}$.

The nonlinear HS dynamics is formulated in terms of the associative star product

$(f * g)(Z ; Y)=\frac{1}{(2 \pi)^{4}} \int d^{4} U d^{4} V \exp \left[i U^{\Lambda} V^{\Omega} C_{\Lambda \Omega}\right] f(Z+U ; Y+U) g(Z-V ; Y+V)$,

where $U^{\Lambda}$ and $V^{\Lambda}$ are spinor integration variables. The star product (25) again yields a particular realization of the Weyl algebra corresponding to the normal ordering with respect to the creation and annihilation operators $B^{\Lambda}=$ $\frac{1}{2}\left(Y^{\Lambda}-Z^{\Lambda}\right)$ and $A_{\Lambda}=\frac{1}{2}\left(Y_{\Lambda}+Z_{\Lambda}\right)$ obeying the commutation relations

$$
\left[A_{\Lambda}, A_{\Omega}\right]_{*}=\left[B^{\Lambda}, B^{\Omega}\right]_{*}=0, \quad\left[A_{\Lambda}, B^{\Omega}\right]_{*}=i \delta_{\Lambda}^{\Omega}
$$

The following simple formulas are true

$$
\left[Y_{\Lambda}, f\right]_{*}=2 i \frac{\partial f}{\partial Y^{\Lambda}}, \quad\left[Z_{\Lambda}, f\right]_{*}=-2 i \frac{\partial f}{\partial Z^{\Lambda}}
$$

for any $f(Z, Y)$. From (25) it follows that functions $f(Y)$ independent of $Z$ form a proper subalgebra with the Weyl star product (8).

The full system of $4 \mathrm{~d}$ equations has the form

$$
\begin{gathered}
d W=W * W, \quad d B=W * B-B * W, \quad d S=W * S-S * W, \\
S * B=B * S, \quad S * S=d Z^{\Omega} d Z^{\Lambda}\left(-i C_{\Omega \Lambda}+4 R_{\Omega \Lambda}(B)\right) .
\end{gathered}
$$


The function $R_{\Omega \Lambda}(B)$ that encodes all information about the HS dynamics has the form

$$
d Z^{\Omega} d Z^{\Lambda} R_{\Omega \Lambda}(B)=\frac{1}{4 i}\left(d z_{\alpha} d z^{\alpha} \mathcal{F}(B) *\left(k e^{i z_{\alpha} y^{\alpha}}\right)+d \bar{z}_{\dot{\alpha}} d \bar{z}^{\dot{\alpha}} \overline{\mathcal{F}}(B) *\left(\bar{k} e^{i \bar{z}_{\dot{\alpha}} \bar{y}^{\dot{\alpha}}}\right)\right)
$$

where $\mathcal{F}(B)$ is some star product power series in $B$ that parametrizes an ambiguity in the HS interactions. Even the simplest choice $\mathcal{F}(B)=B$ leads to the nontrivial (nonlinear) dynamics. The case $\mathcal{F}=0$ leads to the free field equations. Let us note that the equations (29) have the form of the deformed oscillator algebra [37] equivalent to what is sometimes referred in the literature as fuzzy sphere 38 .

The equations (28) and (29) are invariant under the gauge transformations

$$
\delta W=d \varepsilon+[\varepsilon, W]_{*}, \quad \delta S=[\varepsilon, S]_{*}, \quad \delta B=[\varepsilon, B]_{*} .
$$

The space-time differential $d$ only emerges in the equations (28) which have a form of zero-curvature and covariant constancy conditions and therefore admit explicit solution in the pure gauge form

$$
\begin{gathered}
W=-g^{-1}(Z ; Y ; Q \mid x) * d g(Z ; Y ; Q \mid x), \\
B(Z ; Y ; Q \mid x)=g^{-1}(Z ; Y ; Q \mid x) * b(Z ; Y ; Q) * g(Z ; Y ; Q \mid x), \\
S(Z ; Y ; Q \mid x)=g^{-1}(Z ; Y ; Q \mid x) * s(Z ; Y ; Q) * g(Z ; Y ; Q \mid x)
\end{gathered}
$$

with some invertible $g(Z ; Y ; Q \mid x)$ and arbitrary $x$-independent functions $b(Z ; Y ; Q)$ and $s(Z ; Y ; Q)$. Due to the gauge invariance of the whole system one is left with only the equations (29) for $b(Z ; Y ; Q)$ and $s(Z ; Y ; Q)$. These encode in a coordinate independent way all information about the dynamics of massless fields of all spins. In fact, the "constraints" (29) just impose appropriate restrictions on $b$ and $s$ to guarantee that the original space-time equations of motion are satisfied. Let us stress some parallelism between this coordinate-free formulation of the HS dynamics and Matrix formulation of the suprestring theory. From this perspective the equations (29) provide a covariant "matrix" formulation of the HS gauge theory.

A simplest vacuum solution of the equation (29) is $B_{0}=0$ and $S_{0}=d Z^{\Omega} Z_{\Omega}$. From (27) it follows that

$$
\left[S_{0}, f\right]_{*}=-2 i \partial f, \quad \partial=d Z^{\Omega} \frac{\partial}{\partial Z^{\Omega}}
$$


Interpreting the deviation of the full field $S$ from the vacuum value $S_{0}$ as a $Z$-component of the gauge field, $S=S_{0}+2 i d Z^{\Omega} W_{\Omega}$, one rewrites the equations (28), (29) as

$$
\mathcal{R}=d Z^{\Omega} d Z^{\Lambda} R_{\Omega \Lambda}(B), \quad \mathcal{D} B=0
$$

where the generalized curvatures and covariant derivative are defined by the relations

$$
\begin{aligned}
& \mathcal{R}=(d+\partial)\left(d x^{\underline{n}} W_{\underline{n}}+d Z^{\Omega} W_{\Omega}\right)-\left(d x^{\underline{n}} W_{\underline{n}}+d Z^{\Omega} W_{\Omega}\right) \wedge\left(d x^{\underline{n}} W_{\underline{n}}+d Z^{\Omega} W_{\Omega}\right), \\
& \mathcal{D}(A)=(d+\partial) A-\left(d x^{\underline{n}} W_{\underline{n}}+d Z^{\Omega} W_{\Omega}\right) * A+A *\left(d x^{\underline{n}} W_{\underline{n}}+d Z^{\Omega} W_{\Omega}\right) .
\end{aligned}
$$

$\left(d x \underline{\underline{n}} d Z^{\Omega}=-d Z^{\Omega} d x^{\underline{n}}\right.$.) We see that the functions $R_{\Omega \Lambda}(B)$ in (29) identify with the $Z Z$ components of the generalized curvatures, while $x x$ and $x Z$ components of the curvature vanish. The equation $\mathcal{D} B=0$ means that the curvature $R_{\Omega \Lambda}(B)$ is covariantly constant. In fact, it is the compatibility condition for the equations (29), (30) and (37).

The consistency of the system of equations (28), (29) guarantees that it admits a perturbative solution as a system of differential equations with respect to $Z_{\Omega}$. A natural vacuum solution is $W_{0}(Z ; Y ; k, \bar{k} \mid x)=\omega_{0}(Y \mid x), B_{0}=0$ and $S_{0 \Omega}=Z_{\Omega}$ with the field $\omega_{0}(13)$ describing the $A d S_{4}$ vacuum. All fluctuations of the fields can be expressed modulo gauge transformations in terms of the initial data (24) identified with the physical HS fields. Inserting thus obtained expressions into (28) one reconstructs all nonlinear corrections to the free field equations (18), (19).

\section{Towards Unfolded Formulation of the 4d Conformal Higher Spin Theory}

The results discussed so far were established quite some time ago. Now we announce some recent development in the $4 \mathrm{~d}$ conformal HS theory originally proposed by Fradkin and Linetsky [32, 33.

The extension to the conformal case is based on the embedding $o(3,2) \sim$ $s p(4 \mid R) \subset s u(2,2) \sim o(4,2)$. The conformal generators can be realized as the bilinears built from the bosonic oscillators $A_{\Omega}=\left(a_{\alpha}, \bar{a}_{\dot{\alpha}}\right)$ and $B^{\Omega}=\left(b^{\alpha}, \bar{b}^{\dot{\alpha}}\right)$ satisfying the commutation relations (26). The particle number operator $N=-i\left\{A_{\Omega}, B^{\Omega}\right\}$ identifies with the central element of $u(2,2)$. The $4 d$ conformal HS algebra $h s c^{\infty}(4)$ was defined by Fradkin and Linetsky [32] as the subalgebra of the (Lie superalgebra built from the) Weyl algebra spanned by the 
elements having equal numbers of the oscillators $A_{\Omega}$ and $B^{\Omega}$, i.e. $h s c^{\infty}(4)$ is the centralizer of $N$. The HS curvatures have the form (7) with the gauge fields $\omega=\omega(A ; B \mid x)$ and the appropriately modified star product.

Fradkin and Linetsky have shown [33] that there exists a generalization of the $A d S_{4}$ action (15) to the conformal case, which is bilinear in the conformal HS curvatures and gauge invariant at the cubic level. At the free level it contains higher derivatives (the higher spin is the higher derivatives appear) and describes non-unitary dynamics (contains ghosts). Nevertheless, the conformal HS theory may be useful to describe the unitary $A d S_{4}$ HS theory with the help of compensators. It is therefore interesting to study whether the "unfolded" machinery originally developed for the $A d S_{4}$ case can be applied to the conformal HS theory. Here we make a first modest step in this direction formulating unfolded form for the constraints for the extra fields in the $4 \mathrm{~d}$ conformal HS gauge theory.

Let us fix the background 1-form gauge field in the form $\omega_{0}=h^{\alpha}{ }_{\dot{\beta}} a_{\alpha} \bar{b}^{\dot{\beta}}$, where $h_{\dot{\beta}}^{\alpha}$ is the flat Minkowski frame identified with the sigma matrices. (Note that the conformal HS theory admits the flat background not only at the quadratic level but also at the interaction level [33.) Since $h_{\dot{\beta}}^{\alpha}$ can be chosen $x$-independent and $\omega_{0}$ is built from mutually commuting oscillators, the zero curvature equation (12) is trivially satisfied.

As in the $A d S_{4}$ case, the component conformal HS fields in the expansion of the gauge field $\omega$ in powers of the auxiliary spinor variables classify either as dynamical fields satisfying the conformal field equations or as auxiliary and extra fields that express algebraically in terms of the dynamical fields and their derivatives by virtue of some constraints. The dynamical fields are identified with those carrying the lowest conformal dimension with respect to the dilatation generator $D=-i\left(a_{\alpha} b^{\alpha}-\bar{a}_{\dot{\alpha}} b^{\dot{\alpha}}\right)$. Fradkin and Linetsky have suggested the constraints analogous to (17) that express all fields in terms of the dynamical fields and their derivatives. With the aid of these constraints they have shown that the conformal HS action remains gauge invariant in the first nontrivial order in the interactions. A comment we want to make here is that the constraints of the conformal HS gauge theory admit an equivalent unfolded form

$$
\begin{aligned}
R(A, B \mid x) & =h_{\dot{\beta}}^{\alpha} \wedge h^{\gamma \dot{\beta}} \frac{\partial^{2}}{\partial b^{\alpha} \partial b^{\gamma}} C(a, b, \bar{a}, 0 \mid x) \\
& +h_{\gamma \dot{\alpha}} \wedge h_{\dot{\beta}}^{\gamma} \frac{\partial^{2}}{\partial \bar{a}_{\dot{\alpha}} \partial \bar{a}_{\dot{\beta}}} \bar{C}(0, b, \bar{a}, \bar{b} \mid x) .
\end{aligned}
$$

The compatibility conditions of (39) along with free conformal HS equations 
read

$$
\begin{aligned}
& d C(A, B \mid x)=h_{\dot{\beta}}^{\alpha}\left(\frac{\partial^{2}}{\partial b^{\alpha} \partial \bar{b}_{\dot{\beta}}}-a_{\alpha} \frac{\partial}{\partial \bar{a}_{\dot{\beta}}}\right) C(A, B \mid x), \\
& d \bar{C}(A, B \mid x)=h_{\dot{\beta}}^{\alpha}\left(\frac{\partial^{2}}{\partial a^{\alpha} \partial \bar{a}_{\dot{\beta}}}-\bar{b}^{\dot{\beta}} \frac{\partial}{\partial b^{\alpha}}\right) \bar{C}(A, B \mid x) .
\end{aligned}
$$

Here the fields associated with integer spins satisfy $\left(N_{a}+N_{\bar{a}}-N_{b}-N_{\bar{b}}\right) \omega(A, B \mid x)=$ 0 for gauge 1-forms and $\left(N_{a}+N_{\bar{a}}-N_{b}+N_{\bar{b}}+2\right) C(A, B \mid x)=0,\left(N_{a}-N_{\bar{a}}+\right.$ $\left.N_{b}+N_{\bar{b}}+2\right) \bar{C}(A, B \mid x)=0$ for Weyl 0-forms (here $N_{a}=a_{\alpha} \frac{\partial}{\partial a_{\alpha}}, N_{\bar{a}}=\bar{a}_{\dot{\alpha}} \frac{\partial}{\partial \bar{a}_{\dot{\alpha}}}$, $\left.N_{b}=b^{\alpha} \frac{\partial}{\partial b^{\alpha}}, N_{\bar{b}}=\bar{b}^{\dot{\alpha}} \frac{\partial}{\partial b^{\alpha}}\right)$. Note that the part of the equations (39) with negative conformal dimension has the vanishing right hand side. Those with zero conformal dimension contain a definition of the conformal HS Weyl tensors.

The equations (39) and (40) generalize the $A d S_{4}$ equations (18) and (19) to the conformal HS theory. An important difference compared to the $A d S_{4}$ case is that (39) describes the linearized off-mass-shell constraints. A nonlinear deformation of the equations (39) will therefore solve the problem of off-massshell constraints in the conformal HS theory. Hopefully, the development along these lines will lead to the solution of the analogous problem in the $A d S_{4} \mathrm{HS}$ theory via the compensator mechanism, and, eventually, to a full Lagrangian formulation of the both conformal and $A d S_{4} \mathrm{HS}$ theories. An elegant form of the equations (39) and (40) indicates a deep algebraic structure underlying the full conformal HS theory.

\section{Conclusions}

Let us summarize some key features of the HS gauge theories. HS gauge theories are based on the infinite-dimensional HS symmetries [19] realized as the algebras of oscillators carrying spinorial representations of the space-time symmetries [20]. These star product algebras exhibit usual quantum-mechanical nonlocality in the auxiliary spinor spaces. The constraints in the HS gauge theory transform this nonlocality into a space-time nonlocality at the interaction level. The same time the HS gauge theories remain local at the linearized level because the space-time symmetries are realized in terms of bilinears in spinor oscillators. The relevant geometric setting is provided by the Weyl bundle with space-time base manifold and Weyl algebras with spinor generating elements as the fibre. The star product acts in the fiber rather than directly in the space-time. The noncommutative Yang-Mills theory structure also appears in the fiber sector. This is different from the non-commutative 
Yang-Mills theory in the string theory [36]. Another important difference is that some of the ingredients of the theory like twisted adjoint representation used to describe matter fields and HS Weyl tensors do not admit a deformation quantization interpretation. It is interesting to see whether these features of the HS theory will get some counterparts in the string theory.

Finally, let us note that an important analogy with the string theory is that the HS theory is based on the associative (star product) algebras and therefore shares many of the features of the non-commutative geometry. In particular, the whole construction extends [35] to the case with inner symmetries by endowing all fields with the matrix indices. HS gauge theories with nonAbelian symmetries were classified in [39] (where also the systematic notation for the HS algebras was introduced) in a way analogous to the Chan-Paton symmetries for oriented and non-oriented strings.

Acknowledgments. This research was supported in part by INTAS, Grant No.99-1-590 and by the RFBR Grants No.99-02-16207 and 00-15-96566.

[1] P.A.M.Dirac, Proc. R. Soc. A155 (1936) 447.

[2] M.Fierz and W.Pauli, Proc. R. Soc. A173 (1939) 211.

[3] W.Rarita and J. Schwinger, Phys. Rev. 60 (1941) 61.

[4] V.L.Ginzburg and I.E.Tamm, ZhETF 17 (1947) 227.

[5] V.L.Ginzburg, "About Fradkin and the Equations for Higher-spin particles", in Quantum Field Theory and Quantum Statistics, Essays in honour of the sixtieth birthday of E.S.Fradkin, Eds: I.A.Batalin, C.J.Isham and G.A.Vilkovisky, Adam Hilger, Bristol 1987, v2. p. 15.

[6] E.S.Fradkin, "On the theory of particles with higher spins", ZhETF 20 (1950) 27.

[7] D.Z.Freedman, P.van Nieuwenhuizen and S.Ferrara, Phys. Rev. D14 (1976) 912.

[8] S.Deser and B.Zumino, Phys. Lett. B62 (1976) 335.

[9] D.Z. Freedman and A. Das, Nucl. Phys. B120 (1977) 221.

[10] E.S. Fradkin and M.A. Vasiliev, "Model of Supergravity with Minimal Electromagnetic Interaction", Preprint FIAN, N197 (1976). 
[11] C. Fronsdal, Phys. Rev. D18 (1978) 3624.

[12] T. Curtright, Phys. Lett. B85 (1979) 219.

[13] J. Fang and C. Fronsdal, Phys. Rev. D18 (1978) 3630; D22 (1980) 1361.

[14] B. de Wit and D.Z. Freedman, Phys. Rev. D21 (1980) 358.

[15] C. Aragone and S. Deser, Phys. Lett. B86 (1979) 161;

F.A. Berends, J.W. van Holten, P. van Niewenhuizen and B. de Wit, J. Phys. A13 (1980) 1643.

[16] E.S. Fradkin and M.A. Vasiliev, unpublished.

[17] A.Bengtsson, I.Bengtsson and L. Brink, Nucl. Phys. B227 (1983) 31, 41;

A.Bengtsson and I. Bengtsson, Class. Quant. Grav. 3 (1986) 927;

A.Bengtsson, Phys. Lett. B182 (1986) 321; Class. Quant. Grav. 5 (1988) 437.

[18] F.A. Berends, G.J. Burgers and H. van Dam, Z. Phys. C24 (1984) 247;

Nucl. Phys. B260 (1985) 295.

[19] E.S. Fradkin and M.A. Vasiliev, Dokl. Acad. Nauk. 29 (1986) 1100; Ann. of Phys. 177 (1987) 63.

[20] M.A. Vasiliev, Fortschr. Phys. 36 (1988) 33.

[21] A.Chamseddine and P.West, Nucl. Phys. B129 (1977) 39;

K. Stelle and P. West, Phys. Rev. D21 (1980) 1466.

[22] S. W. MacDowell and F. Mansouri, Phys. Rev. Lett. 38 (1977) 739.

[23] M. A. Vasiliev, Fortschr. Phys. 35 (1987) 741.

[24] V.E. Lopatin and M.A. Vasiliev, Mod. Phys. Lett. A3 (1988) 257;

M.A. Vasiliev, Nucl. Phys. B301 (1988) 26.

[25] F.A. Berezin, Mat. Sbornik 86 (1971) 578;

F.A. Berezin and M.S. Marinov, Ann. of Phys. 104 (1977) 336;

F. Bayen, M. Flato, C. Fronsdal, A. Lichnerowicz and D. Sternheimer, Ann. of Phys. 110 (1978) 61, 111.

[26] J.E. Moyal, Proc. Cambridge Phil. Soc. 45 (1949) 99. 
[27] F.Berends, G.Burgers and H. van Dam, Nucl. Phys. B271 (1986) 429; D. Anselmi, Theory of Higher Spin Tensor Currents and Central Charges, hep-th/9808004; Class. Quant.Grav. 17 (2000) 1383 hep-th/9906167.

[28] M.Vasiliev, Contributed article to Golfand's Memorial Volume "Many faces of the superworld", ed. by M.Shifman, World Scientific Publishing Co Pte Ltd, Singapore, 2000; hep-th/9910096.

[29] E. S. Fradkin and M. A. Vasiliev, Phys. Lett. B189 (1987) 89; Nucl. Phys. B291 (1987) 141.

[30] M.A. Vasiliev, Phys. Lett. B243 (1990) 378; Class. Quant. Grav. 8 (1991) 1387; Phys. Lett. B285 (1992) 225.

[31] E.S. Fradkin and R.R. Metsaev, Class. Quant. Grav. 5 (1991) L89;

R.R. Metsaev, Mod. Phys. Lett. A6 (1991) 359, 2411; ibid A8 (1993) 2413; Class. Quant. Grav. 10 (1993) L39; Phys. Lett. B309 (1993) 39.

[32] E.S. Fradkin and V.Ya. Linetsky, Ann. of Phys. 198 (1990) 252.

[33] E.S. Fradkin and V.Ya. Linetsky, Phys. Lett. B 285 (1989) 97.

[34] E.S. Fradkin and A.A.Tseytlin, Phys. Rep. 119 (1985) 233.

[35] M.A. Vasiliev, Ann. Phys. (N.Y.) 190 (1989) 59.

[36] A. Connes, M.R. Douglas and A. Schwarz, JHEP 02 (1998) 003; hepth/9711162;

M. Douglas and C. Hull, JHEP 02 (1998) 8; hep-th/9711165;

Chong-Sun Chu, Pei-Ming Ho, Nucl. Phys. B 550, 151 (1999), hepth/9812219; hep-th/9906192;

V. Schomerus, "D-branes and Deformation Quantization" hep-th/9903205;

N. Seiberg and E. Witten, JHEP 09 (1999) 032, hep-th/9908142.

[37] E. Wigner, Phys. Rev. 77 (1950) 711;

L. M.Yang, Phys. Rev. 84 (1951) 788;

D. G. Boulware and S. Deser, Il Nouvo Cimento, XXX (1963) 231;

M.A. Vasiliev, JETP Lett. 50 (1989) 374; Int. J. Mod. Phys. A6 (1991) 1115 .

[38] J.Madore, Class. Quant. Grav. 9 (1992) 69.

[39] S.E. Konstein and M.A. Vasiliev, Nucl. Phys. B 331 (1990) 475. 